\title{
Multiphase Turbulent Interstellar Medium: Some Recent Results from Radio Astronomy
}

NIRUPAM ROY*\#

Max-Planck-Institut für Radioastronomie MPIfR, Auf dem Hügel 69, Bonn, D-53121, Germany

(Received on 4 May 2014; Revised on 21 January 2015; Accepted on 5 February 2015)

\begin{abstract}
The radio frequency $1.4 \mathrm{GHz}$ transition of the atomic hydrogen is one of the important tracers of the diffuse neutral interstellar medium. Radio astronomical observations of this transition, using either a single dish telescope or an array interferometer, reveal different properties of the interstellar medium. Such observations are particularly useful to study the multiphase nature and turbulence in the interstellar gas. Observations with multiple radio telescopes have recently been used to study these two closely related aspects in greater detail. This review article presents a brief outline of some of the basic ideas of radio astronomical observations and data analysis, summarizes the results from these recent observations, and discusses possible implications of the results. Using various observational techniques, the density and the velocity fluctuations in the Galactic interstellar medium was found to have a Kolmogorov-like power law power spectra. The observed power law scaling of the turbulent velocity dispersion with the length scale can be used to derive the true temperature distribution of the medium. Observations from a large ongoing atomic hydrogen absorption line survey have also been used to study the distribution of gas at different temperature. The thermal steady state model predicts that the multiphase neutral gas will exist in cold and warm phase with temperature below $200 \mathrm{~K}$ and above $5000 \mathrm{~K}$ respectively. However, these observations clearly show the presence of a large fraction of gas in the intermediate unstable phase. These results raise serious doubt about the validity of the standard model, and highlight the necessity of alternative theoretical models. Interestingly, numerical simulations suggest that some of the observational results can be explained consistently by including the effects of turbulence in the models of the multiphase medium.
\end{abstract}

Key Words: ISM: atoms; - ISM: general; ISM: structures; Radio Lines; ISM: turbulence

\section{Introduction}

The interstellar space in a galaxy is far from empty. Even if the density is very low $\left(\sim 10^{-4}-10^{6} \mathrm{~cm}^{-3}\right)$, the region between the stars is filled with the interstellar medium (ISM) consisting of gas, dust, charged particles and magnetic fields. Astronomers inferred the existence of interstellar gas after the discovery of stationary absorption lines of ionized calcium (Ca II) towards the spectroscopic binary $\delta$ Orionis (Hartmann, 1904). Any spectral line from stars of a spectroscopic binary system should have a periodic wavelength shift due to the Doppler effect for the motion of the stars. The stationary Ca II absorption line was hence taken as an indication of interstellar gas. Edward Emerson Barnard first carried out a systematic study of the Galactic ISM, and proposed the existence of "intervening opaque masses" between the stars to explain "dark nebula". He used his expertise of astrophotography to produce the first images of dark nebulae and published the first catalogue of such dark clouds (Barnard, 1919). Over time, further imaging and spectroscopic observations, made the existence of widespread ISM, including gas and dust clouds, quite evident.

*Author for Correspondence: E-mail: nroy@ mpifr.de; Tel: +49 288 525-491 Fax: +49 228 525-229

\#Present Address: Department of Physics, Indian Institute of Technology-Kharagpur, Kharagpur 721 302, India 
It is now well established that the process of star formation through the collapse of protostellar clouds is never totally efficient. This leads to the existence of residual gas around the stars. The radiation and mechanical energy inputs from the stars, in turn, influence the properties of the ISM. Processes like stellar winds, episodic ejection of mass during the stellar evolution and supernova explosions transfer material and energy from the stars to the ISM. Similarly, ultraviolet radiation from hot, young stars and cosmic rays heat and ionize the ISM. Due to all these processes, the ISM is strongly coupled to the stars in a galaxy, and play a crucial role between the stellar and the galactic scales. Hence, understanding the properties of the ISM is very important from many considerations in astrophysics.

Multiphase nature and turbulence are two of the key ingredients of the ISM physics. In the standard model, multiple phases of the ISM, with different densities, temperatures and ionization states, coexist in rough thermal pressure equilibrium (e.g. Field, 1965; Field et al., 1969). In a multiphase medium, the cold dense neutral gas (cold neutral medium; CNM) is embedded in either neutral or ionized low density warm gas (warm neutral and ionized medium; WNM/ WIM), which again may be embedded in much lower density hot ionized medium (HIM). Considering different cooling and heating processes, it can be shown that the neutral-hydrogen gas (H I) can be in thermal steady state in one of the two stable ranges of temperature: (i) 40 - 200 K for CNM and (ii) $5000-8000 \mathrm{~K}$ for WNM. The H I at intermediate temperatures is unstable, and, due to runaway heating or cooling, moves to either cold or warm phase (McKee and Ostriker, 1977; Wolfire et al., 1995, 2003). The ISM (of the Milky Way as well as of other galaxies) is also known to have a clumpy, self-similar, hierarchical structure over several orders of magnitude in scale (Larson, 1981; Falgarone et al., 1992; Heithausen, et al., 1998). Various direct and indirect observations suggest that these structures extend down to $\lesssim 10$ AU scales (e.g. Hill et al., 2005; Dutta et al., 2014). On the theoretical front, the ISM is known to be turbulent, and hence is expected to exhibit such density structures and velocity fluctuations over a wide range of scales (Crovisier and Dickey, 1983; Green, 1993; Lazarian and Pogosyan, 2000). However, the nature of the turbulence in different phases of the ISM is not yet understood properly.

This article presents an overview of some recent results, from radio astronomical observations, which have important implications for our current understanding of the multiphase turbulent ISM. A significant fraction of the observations, on which these results are based, were carried out with the Giant Metrewave Radio Telescope (GMRT; Swarup et al., 1991), a world-class radio telescope located near Pune, Maharashtra, in India. A few important aspects of the observational techniques in radio astronomy, and a brief overview of the GMRT are outlined in the "Observation and Analysis" section. The results, related to the ISM turbulence and the multiphase nature, are summarized in sections titled "Turbulence in the diffuse neutral ISM" and "Temperature of the diffuse neutral ISM" respectively. Finally, a discussion on the broader aspects of these results, and on possible future directions are presented in the "Discussions and Conclusions" section.

\section{Observation and Analysis}

Historically, astronomy was confined to observations of the sky mostly with optical telescopes, probing only

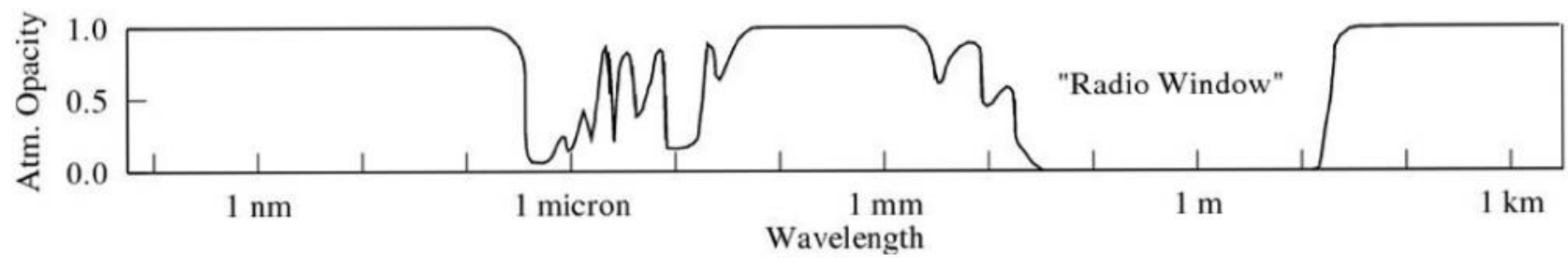

Fig. 1: A toy model of atmospheric opacity to electromagnetic radiation at different wavelength. Note the low opacity for optical $(\lambda \sim 400-700 \mathrm{~nm})$ and radio waves (few $\mathrm{cm}$ to $\sim 10 \mathrm{~m}$ ) 
a narrow window of visible lights of the whole electromagnetic spectrum. With technological progress and developments, today astronomers are, however, capable of observing, using ground- and space-based telescopes, across the whole spectrum $-\gamma$-rays, $X$-rays, ultraviolet, visible, infrared, microwave and radio emission originating in different astrophysical processes from a variety of sources. Due to low or negligible atmospheric opacity at the radio "window" $(\lambda \approx$ a few $\mathrm{cm}$ to about $10 \mathrm{~m}$; see Fig. 1), observations at these wavelengths are possible to carry out from ground-based telescopes.

Radio astronomy emerged as a subject in the first half of the last century, with the pioneering work of Karl Guthe Jansky, who first reported the detection of celestial radio signal (Jansky, 1933). His contribution has been recognised in many ways from naming the unit of flux density Jansky $\left(1 \mathrm{Jy}=10^{-26} \mathrm{~W} \mathrm{~m}^{-2} \mathrm{~Hz}^{-1}\right)$, to recent renaming of the upgraded radio telescope as the Karl G. Jansky Very Large Array (VLA). Over time, radio observations have revealed thermal and non-thermal emission from known as well as new classes of sources including the Sun, supernova remnants, pulsars, regions of ionized hydrogen (H II regions) associated with star formation, active galactic nuclei, radio galaxies and clusters of galaxies. To get an overview of the exciting results from radio astronomical studies, see Burke and Graham-Smith (2014). For the purpose of this article, let us now consider a very important radio frequency transition, the famous H I $21 \mathrm{~cm}$ line, in greater detail.

\section{Atomic Hydrogen $21 \mathrm{~cm}$ Radiation}

In the radio frequencies, the most useful tracer of the ISM is the H I $1.4 \mathrm{GHz}(21 \mathrm{~cm})$ line. This is not only because hydrogen is the most abundant element in the ISM, but also because it is possible to extract a lot of useful information (e.g. temperature, column density, velocity, magnetic field strength) about the physical properties of the ISM from H I $21 \mathrm{~cm}$ observations. In 1944, Hendrik van de Hulst predicted the possibility of detecting $\mathrm{H} \mathrm{I} 21 \mathrm{~cm}$ radiation of celestial origin (van de Hulst, 1945), and it was first observed by Ewen and Purcell (1951). This line emission (or absorption) is caused by the transition between the two hyperfine states of the $1^{2} S_{\frac{1}{2}}$ ground state of hydrogen. These two states with parallel and anti-parallel electron/proton spin configuration (i.e. total spin angular momentum $\mathrm{F}=1$ and 0 ) have slightly different energy (see Fig. 2). The energy difference $\mathrm{h} u$ corresponds to the frequency of the transition $v_{10}$ $=1420.405752 \mathrm{MHz}(\sim 21.1 \mathrm{~cm})$.

The $21 \mathrm{~cm}$ transition of neutral hydrogen has an extremely small probability of $2.9 \times 10^{-15} \mathrm{~s}^{-1}$. So, the natural lifetime of the excited state for a single isolated atom is about 10 million years. But, because of the processes like collisions and interaction with the background radiation field, the lifetime can be considerably shortened. This, and the fact that the total number of atoms in the ISM is quite large, make the $21 \mathrm{~cm}$ emission (or absorption against a background source) readily detectable.

In thermodynamic equilibrium, the number density of atoms in the upper and lower energy states are related by the Boltzmann distribution, $n_{1}=3 n_{0}$ $\exp \left(-h v_{10} / k T\right)$. Even if the gas is not in thermodynamic equilibrium, one can define a characteristic temperature, called the spin temperature $\left(T_{s}\right)$, that satisfies the observed distribution between the two spin states.

Either collision or radiative mechanisms may cause direct transition between the two hyperfine states. Alternatively, higher energy levels of hydrogen atom can be excited from one of the hyperfine states, followed by de-excitation to the other hyperfine state via Ly- $\alpha$ or higher Lyman lines transitions (see Fig. 2 ). The probability of transition via Ly- $\alpha$ photons depends on the intensity profile of radiation near the Ly- $\alpha$ frequency. Radiation which has been scattered many times in the ISM will have a profile dependent on the kinetic temperature $\left(T_{k}\right)$ of the gas. This may tend to couple $T_{s}$ to $T_{k}$, particularly in low density gas. In high density regions, the collisional mechanism dominates, and may similarly couple $T_{s}$ to $T_{k}$ (Field, 1958). Whether or not $T_{s}$ is same as $T_{k}$, for a constant $T_{s}$ along the line of sight, it can be shown (Kulkarni and Heiles, 1988) that $T_{B}(v)=T_{s}\left[1-e^{-\tau(v)}\right]$, where 


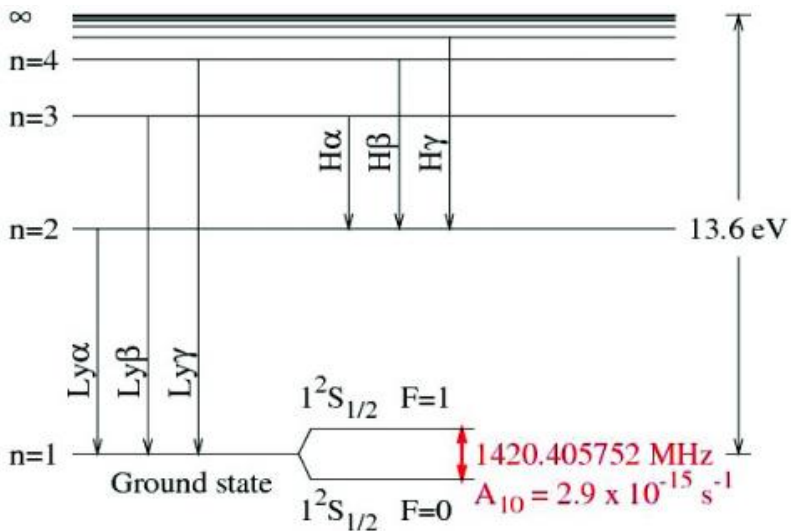

Fig. 2: A simplified representation of the energy levels of atomic hydrogen showing the hyperfine splitting of the ground state. $L y-\alpha$ and other higher energy level transitions may play a role in bringing equilibrium between the hyperfine states

$T_{B}(v)=I(v) c^{2} / 2 k v^{2}$ is the observed brightness temperature, $\tau(v)$ is the optical depth at frequency $v, I(v)$ is the intensity, $k$ is the Boltzmann constant and $\tau$ is the speed of light. At low optical depth limit, the spin temperature will be $T_{s}(v) \approx T_{B}(v) / \tau(v)$.

\section{Radio Telescopes}

Though there may be considerable varieties (see, e.g., LOFAR and ORT in Fig. 3), radio telescopes are generally parabolic antennas to receive and detect radio frequency electromagnetic waves. A radio telescope may consist of either a single element ("single dish" telescope), or multiple elements ("array" telescope). The technique of interferometry is routinely used in radio astronomy to achieve better sensitivity and angular resolution or the "beam" by combining signals from widely separated elements of an array telescope. The resolution of a single dish telescope is $\approx \lambda / D$, while the same for an interferometer is $\approx \lambda / B$ for an observing wavelength of $\lambda$, dish diametre $D$ and maximum separation between antennas $B$ (known as interferometer "baseline"). A single dish telescope is sensitive to the total intensity $I(l, m)$ towards the direction of observation $(l, m)$ on the sky. An interferometer, on the other hand, directly measures the Fourier transform of $I(l, m)$,

$$
V(u, v)=\iint I(l, m) e^{-2 \pi i(u l+v m)} d l d m .
$$

Each combination of antenna pair sample the real and the imaginary part (or amplitude and phase) of the visibility function $V(u, v)$ at an inverse angular separation $(u, v)$ corresponding to projected baseline separation perpendicular to the source direction. As an example, Fig. 4A and 4B show the calibrated visibility data for a GMRT $610 \mathrm{MHz}$ observation. At a given time, an array with $N$ elements sample the visibility function on $N(N-1) / 2$ points on the $(u, v)$ plane. During the course of the observation, as the apparent position of the source on the sky changes with time, the projected baseline separation also changes. This technique of sampling the $(u, v)$ plane is known as the aperture synthesis. The maximum baseline for such arrays may be from few $\mathrm{km}$ to tens of $\mathrm{km}$, and even few thousand $\mathrm{km}$ for very long baseline interferometry (VLBI). The longest baseline of the Very Long Baseline Array (VLBA) of the National Radio Astronomy Observatory (NRAO), for example, is $\sim 8600 \mathrm{~km}$ (Mauna Kea, Hawaii to St. Craix, Virgin Islands). Inverse Fourier transform of $V(u, v)$ is used to make the "radio image" of the sky. Fig. 4C also shows the corresponding image created using the Astronomical Image Processing Software (AIPS). Details of methods for either single dish or interferometric observations, and standard data analysis procedures are beyond the scope of this article. One may see Thompson et al. (2001), Wilson et al. (2013), and references therein, for a comprehensive discussion on these topics.

\section{The Giant Metrewave Radio Telescope}

The GMRT is, at present, world's largest low frequency radio telescope. It is located near the village Khodad in Maharashtra, approximately $80 \mathrm{~km}$ from Pune. The GMRT consists of 30 parabolic dishes, each with a diametre of $45 \mathrm{~m}$. The telescope operates at $\sim 150,235,325,610$ and $1420 \mathrm{MHz}$. Half of the antennas are placed within central $1 \mathrm{~km} \times 1 \mathrm{~km}$ area, and the rest are placed in roughly a "Y" shaped array (with largest baseline separation of $\sim 30 \mathrm{~km}$ ) for optimum sampling of the $(u, v)$ Fourier domain. The unique feature of GMRT, apart from its high angular 


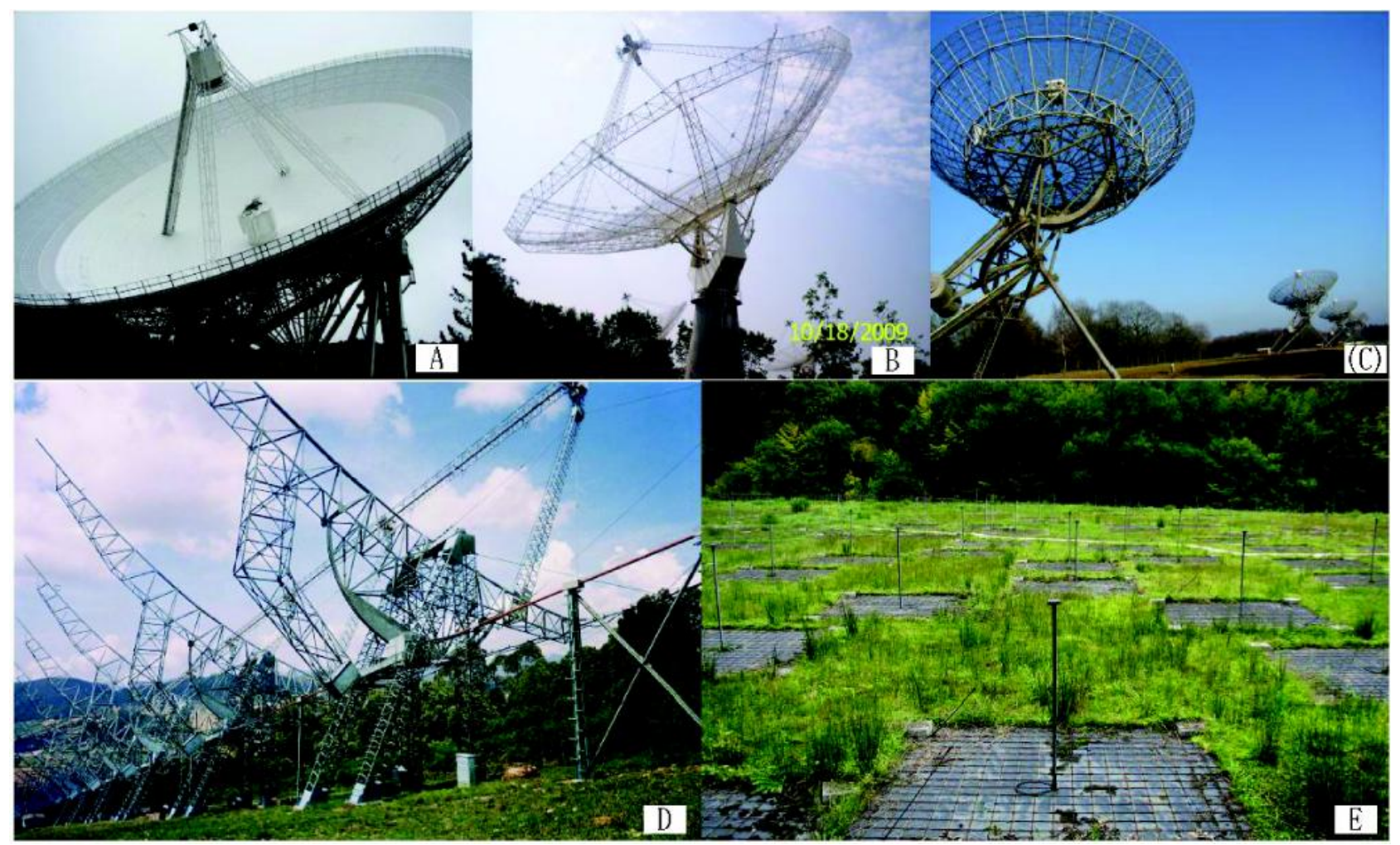

Fig. 3: (A): $100 \mathrm{~m}$ diametre single dish Effelsberg Radio Telescope in Germany (B): One of the 30 dishes of the GMRT array (C): A few of the $25 \mathrm{~m}$ dishes of the Westerbork Synthesis Radio Telescope (WSRT; a 14 element $2.7 \mathrm{~km}$ East-West array) in the Netherlands. Not all radio telescopes are dish shaped. (D): The Ooty Radio Telescope (ORT) is a $530 \mathrm{~m} \times 30 \mathrm{~m}$ cylindrical paraboloid on equatorial mount; (E): Part of a Low Frequency Array (LOFAR) station in Germany consists of the low band dipole antennas (right). See text in the "Radio Telescope" sub-section for details

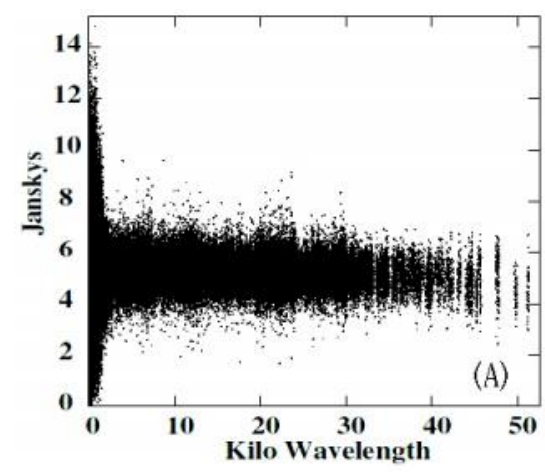

A

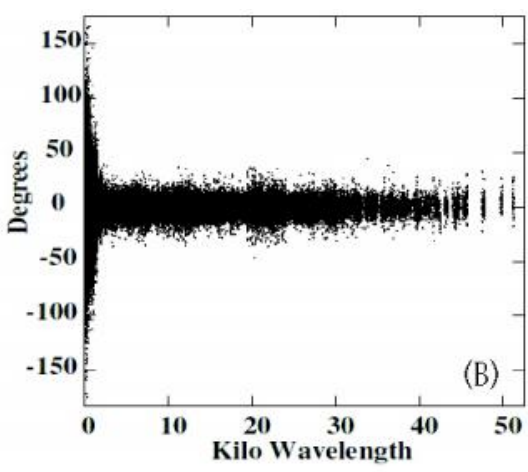

C

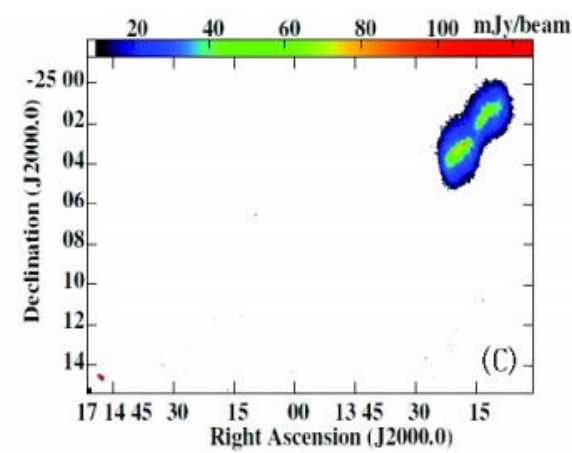

B

Fig. 4: Calibrated visibility data and image from the GMRT $610 \mathrm{MHz}$ observation of the field containing the bright unresolved source NVSSJ171447 - 251435. (A) : Visibility amplitudes and (B): phases as a function of baseline separation; (C): Image of a part of the field, created by inverse Fourier transform of the data, shows a double-lobed radio galaxy at the top right corner, and the point source with $\sim 5$ Jy flux density at the bottom left corner. Note that the flux density scale of the image is intentionally saturated at $\sim 125 \mathrm{mJy}$ to take care of the large dynamic range, and properly display both the bright point source and the faint extended radio galaxy

resolution and low frequency capabilities, is the simultaneous sensitivity of the array to both large and small scale structures due to this hybrid array design. The parabolic surface of the antennae consist of wire mesh which is good reflector at the operating frequency range of the GMRT. All the antennas can be moved to point at different directions in the sky using an altitude-azimuth mounting system. The 
signals from all the antennas are transmitted to the array control building using optical fiber network, and combined in the correlator system to record the visibilities in a digital format. The angular resolution of the GMRT at $1.4 \mathrm{GHz}$ is $\lesssim 2$ arcsecond. It is possible to easily achieve a spectral resolution of $<2$ $\mathrm{kHz}$ per channel $\left(\sim 0.4 \mathrm{~km} \mathrm{~s}^{-1}\right)$. More details on GMRT, including all technical details are summarized by Swarup et al. (1991).

\section{Emission and Absorption Spectra}

A single dish radio telescope with receiver covering the $21 \mathrm{~cm}$ line ("L band") can be used to observe radio emission from the Galactic H I. Such observations typically have coarse angular resolution (tens of arcminute beam size). One may also use a single dish telescope to observe $\mathrm{H}$ I in absorption towards background continuum sources. However, to do that, one must carefully model the emission spectra from observations along adjacent lines of sight, and subtract the contamination from emission within the beam to estimate the absorption spectra. Thus, effectively one assumes that the $\mathrm{H}$ I emission is smooth over the scale of a few beam size. This assumption may not necessarily be correct. A much reliable way to get the absorption spectra is to carry out interferometric observations. With the typical resolution of few arc- second, the diffuse $\mathrm{HI}$ emission is resolved out, and one gets uncontaminated absorption spectra towards background continuum sources. Following standard data reduction steps (removing bad data and radio frequency interference, calibrating instrumental and atmospheric effects, imaging of the visibility data using inverse Fourier transform, converting absorption intensity to optical depth $\tau$ etc.) one finally gets both the $\mathrm{H} \mathrm{I}$ emission and absorption spectra $T_{B}(v)$ and $\tau(v)$ from single dish and/or interferometric observations, with certain spectral resolution $\Delta v$. These spectra are also often expressed as a function of physically more meaningful Doppler velocity $(v)$, instead of observed frequency $(v)$.

\section{Derived Physical Quantities}

For an isothermal $\mathrm{H}$ I cloud, both the emission and the absorption spectra can be modelled as Gaussian profile along the velocity axis. The central velocity of the Gaussian represents the line of sight component of the "bulk" velocity, and is closely related to the Galactic rotation. The velocity width of the line has contribution from thermal and non-thermal velocity dispersion. In absence of any non-thermal motion, the line width provides $T_{k}=\left(m_{H} / k\right)\left(\sigma / 1 \mathrm{~km} \mathrm{~s}^{-1}\right)^{2} \mathrm{~K}$. In reality, however, due to non-thermal broadening, it only provides an upper limit $\left(T_{k, \max }\right)$ of the kinetic temperature. The H I column density (i.e. the integrated number density along the line of sight) is given by,

$$
\begin{aligned}
& N(\mathrm{HI})=1.823 \times 10^{18} T_{s} \\
& \int \tau(v) d v \approx 1.823 \times 10^{18} \int T_{B}(v) d v
\end{aligned}
$$

where $N(\mathrm{H} \mathrm{I})$ is in $\mathrm{cm}^{-2}$ and $v$ is in $\mathrm{km} \mathrm{s}^{-1}$ (Kulkarni and Heiles, 1988; Dickey and Lockman, 1990). The spin temperature, $T_{s}$ is given by,

$$
T_{s}=\frac{N(\mathrm{HI})}{1.823 \times 10^{18} \int \tau(v) d v} \approx \frac{\int T_{B}(v) d v}{\int \tau(v) d v}
$$

where $\tau(v)$ is generally determined from $\mathrm{H} \mathrm{I}$ absorption spectrum towards a background source, and $N(\mathrm{HI})$ is determined from the emission spectrum for a nearby line of sight.

In practice, if the gas is not homogeneous (i.e. having variation of density and temperature, along and transverse to the line of sight), or the optical depths are large $(\tau \gtrsim 1)$, Equation 3 can not be used to determine 'the' spin temperature easily. For example, multiple optically thin $(\tau \lesssim 1)$ components along the line of sight will only give a column- density-weighted harmonic mean temperature of the individual components. In general, there may not be any unique interpretation of the data, although modelling the spectra with many Gaussian components (corresponding to multiple "clouds") is common in such situations (e.g., Mebold et al., 1982; Heiles and Troland, 2003a; Heiles and Troland, 2003b). For extreme situations with very complicated profiles, 
where multi-Gaussian fit may be highly non-trivial and degenerate, one may still extract some useful information by computing $T_{s}(v)=T_{B}(v) / \tau(v)$ for individual channels. Note that the analysis method outlined above can be extended easily for imaging widespread emission or absorption against extended background sources. In those cases, $\tau(v)$ and $T_{B}$ (v) will also be functions of sky coordinates $(l, m)$.

Fig. 5 shows an example of $\mathrm{H}$ I absorption and emission spectra towards quasar PKS 1245-19, a radio bright, unresolved extragalactic source $\left(l=302^{\circ}\right.$, $b=+43^{\circ} .5$ in the Galactic coordinate system). The emission spectrum is taken from the Leiden/Argentine/ Bonn Survey (LAB; Hartmann and Burton, 1997; Bajaja et al., 2005; Kalberla et al., 2005), and the absorption spectrum is from the GMRT. One can clearly see multiple emission and absorption components at different velocity $\left(v_{\mathrm{LSR}}\right)$ with respect to the local standard-of-rest (LSR) frame. The H I column density, $N(\mathrm{H} \mathrm{I})$, computed from the integral of the emission profile, is $\sim 3.7 \times 10^{20} \mathrm{~cm}^{-2}$, and the

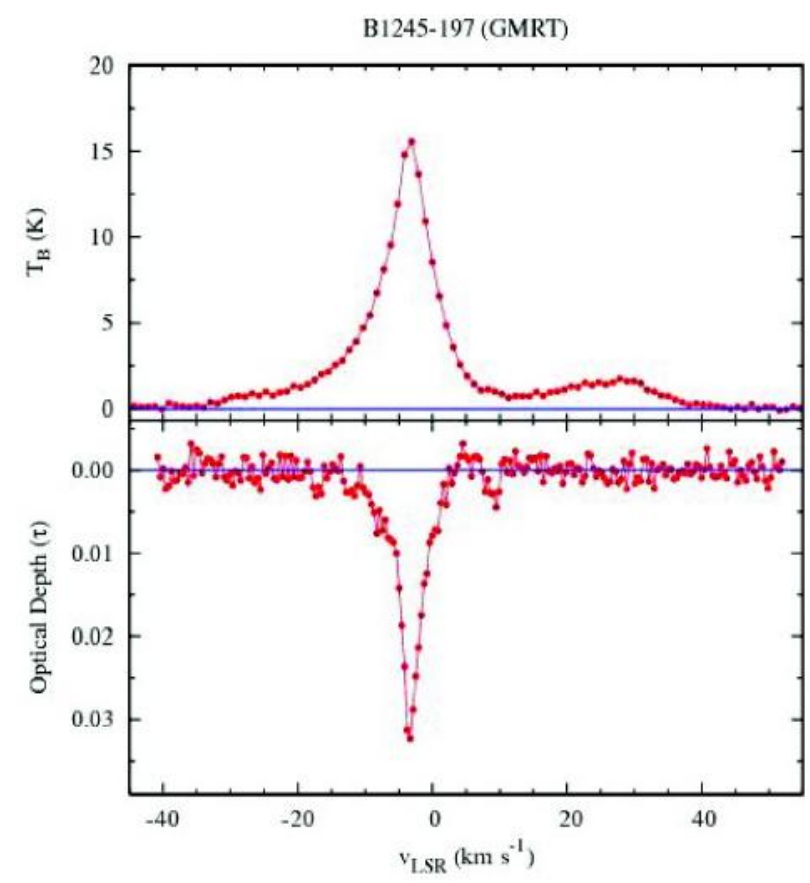

Fig. 5: Profiles of the observed $H$ I $21 \mathrm{~cm}$ emission and absorption towards $l=302^{\circ}, b=+43^{\circ} .5$. The emission spectrum is taken from the LAB survey, and the absorption spectrum obtained with the GMRT (also see the section "Temperature of the diffuse neutral ISM") average spin temperature is $\left\langle T_{s}\right\rangle=1288 \pm 41 \mathrm{~K}$. Both the emission and absorption spectra can also be modelled as a set of multiple Gaussian components. There are some broad emission components without corresponding absorption components. Such components are believed to arise from higher temperature gas (i.e. WNM), with optical depth lower than the detection limit of the observation (Radhakrishnan et al., 1972; Heiles and Troland, 2003a; Heiles and Troland, 2003b). For this particular line of sight, from $\left\langle T_{s}\right\rangle$, and $T_{k, \max }$ values derived from the line widths (Roy et al., 2013b), one can conclude that only $\lesssim 16 \%$ of H I is in the CNM phase (with $T_{s}$ $\lesssim 200 \mathrm{~K})$.

\section{Turbulence in the Diffuse Neutral ISM}

Indications of turbulence in the diffuse neutral ISM come mainly from observations of small scale fluctuations of $\mathrm{H}$ I emission as well as of optical depth. Recently, it has been clearly shown from an ongoing H I absorption survey (Roy et al., 2013a; Ray et al., 2013b, also see the section titled "Temperature of the diffuse neutral ISM") that, for the diffuse H I, the non-thermal broadening contributes significantly (i.e. comparable to the thermal broadening) to the observed line width. There are direct observational evidences of AU scale (milliarc angular scale) structures in H I opacity fluctuations from VLBI observations (Brogan et al., 2005; Roy et al., 2012; Dutta et al., 2014), and also from variation of absorption towards high proper motion pulsars detected using multiepoch observations (Frail et al., 1994). Deshpande et al. (2000) reported scale-free opacity fluctuations at pc and sub-pc scales using interferometric observations towards extended sources. Similarly, H I emission studies have revealed scale-free structures of the neutral ISM in the Milky Way and other nearby galaxies (e.g. Crovisier and Dickey, 1983; Green, 1993; Westpfahl et al., 1999; Dutta et al., 2009). Most of these studies use second order statistics (e.g. structure function, power spectrum) of intensity and opacity fluctuations, and compare those with analytical or numerical models (e.g. Kolmogorov, 1941; Irosnikov, 1964; Kraichna, 1965; Goldreich and Sridhar, 1995) to constrain the nature of the turbulence. 
Power spectra of intensity or opacity fluctuations are related directly to the underlying density fluctuations, and also get affected by the velocity fluctuations (e.g. Lazarian and Pogosyan, 2000). The power spectrum, which is the Fourier transform of the autocorrelation function, is given by

$$
P(\mu, v)=\iint \xi(l, m) e^{-2 \pi i(u l+v m)} d l d m
$$

where $\xi(l, m)$ is the autocorrelation function of the quantity of interest (intensity, opacity, density or velocity fluctuations). The function $\xi(l, m)$ is also closely related to the structure function $S(l, m)$. For the intensity fluctuation, $\xi$ and $S$ can be written as

$$
\begin{aligned}
& \xi\left(l-l^{\prime}, m-m^{\prime}\right)=\left\langle\delta I(l, m) \delta I\left(l^{\prime}, m^{\prime}\right)\right\rangle \\
& S\left(l-l^{\prime}, m-m^{\prime}\right)=\left\langle\left[I(l, m)-I\left(l^{\prime}, m^{\prime}\right)\right]^{2}\right\rangle .
\end{aligned}
$$

Here, angular brackets denote ensemble average over possible realization. If one assumes statistical isotropy, then $P(u, v)$ will be a function of only the magnitude $U=\sqrt{u^{2}+v^{2}}$. It is important to note that, due to the scale free nature of the turbulence, the power spectrum is expected to have a power law form $P(U)=A U^{\alpha}$ over the inertial range of scales. The structure function then will also be a power law (Lee and Jokipii, 1975), with power law index $\beta=-$ $(\alpha+2)$.

One advantage of interferometric observations is that the observed visibilities are already measurements of Fourier transform components of the sky brightness. So, one can estimate the power spectrum from the observed visibilities without going into the complications and limitations of the imaging and deconvolution algorithms (Crovisier and Dickey, 1983; Green, 1993; Bharadwaj and Sethi, 2001).

Based on this principle, a suitable method has been developed for radio interferometers like the GMRT (Roy et al., 2009), and is used to estimate, directly from the visibility data, the H I opacity fluctuations power spectrum towards the extended

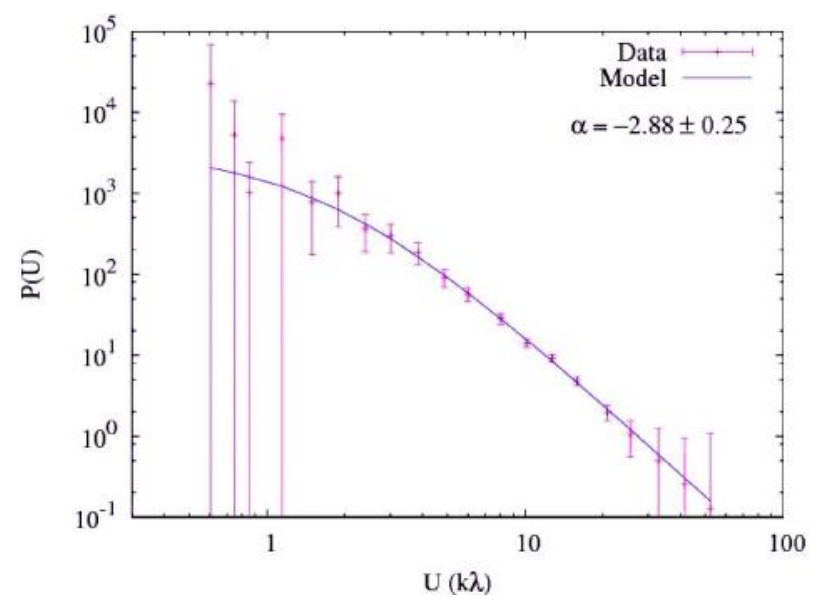

Fig. 6: Estimated intensity fluctuations power spectrum for one spectral channel using visibility data from the GMRT observation of $\mathrm{H} I$ absorption towards Cas $\mathrm{A}$. The solid line is the best-fit model with a power law power spectrum for the opacity fluctuations

background emission from the supernova remnant Cassiopeia A(Cas A). Fig. 6 shows the derived power spectrum for one of the velocity channels, and the best fit model overlaid on the data. It was established that the H I opacity fluctuations have a power law power spectrum with $\alpha=-2.86 \pm 0.10(3 \sigma)$ over the scale of $\sim 500$ AU to $2.5 \mathrm{pc}$ (Roy et al., 2010). The line of sight towards Cas A probes deep absorption from the local arm and the Perseus arm of the Milky Way. There was no significant difference in $\alpha$ for these two spiral arms. The results are in good agreement with other studies suggesting $\alpha \sim-2.7$ to -3 for the diffuse neutral medium (e.g. Crovisier and Dickey, 1983; Deshpande et al., 2000; Dutta et al., 2009). Although the power law behaviour suggests a Kolmogorov-like turbulence, the power law index is significantly shallower than the Kolmogorov $-11 / 3$ spectrum for incompressible turbulence (Kolmogorov, 1941). This mismatch may be due to the fact that even small ionization fraction can couple the gas with the interstellar magnetic field. In that case, the turbulence will be magnetohydrodynamic (MHD) in nature, and the density fluctuation power spectrum may have a different power law index (see Roy et $a l ., 2009$, and references therein for a detailed discussion).

Apart from the density fluctuations, these 
data were also used to constrain the velocity fluctuations using "velocity channel analysis" (Lazarian and Pogosyan, 2000). However, the effect of the velocity width of the spectral channels was found to be insignificant on the power law index. This result only puts a weak constrain on the velocity structure function index $\beta=0.2 \pm 0.6$, broadly consistent with Kolmogorov (1941) prediction of $\beta=2 / 3$.

It is worth mentioning here that this study also revealed the presence of MHD turbulence in the supernova remnant from the continuum image of the background source Cas A. This is revealed by the power spectra of the synchrotron radiation intensity fluctuations derived from the GMRT $1.4 \mathrm{GHz}$ observation of Cas A (see Fig. 7), and also from the VLA $5 \mathrm{GHz}$ archival data for Cas A and Crab Nebula. For Crab Nebula, the power law index is $\approx-3.23$ over the whole range of $U$ probed in these studies. For Cas A, the power spectrum has the same slope at large $U$. However, below $\sim 10 \mathrm{k} \lambda$, there is a break in the spectrum, and the index changes to -2.22 . This break and flattening below $10 \mathrm{k} \lambda$ ( 25 arcsec; an angular scale that corresponds to the shell thickness of Cas A) is interpreted as a 3-D to 2-D transition of the turbulence in the supernova shell (Roy et al., 2009).

Another way to get a handle on the nature of turbulence is to study the scaling of turbulent velocity

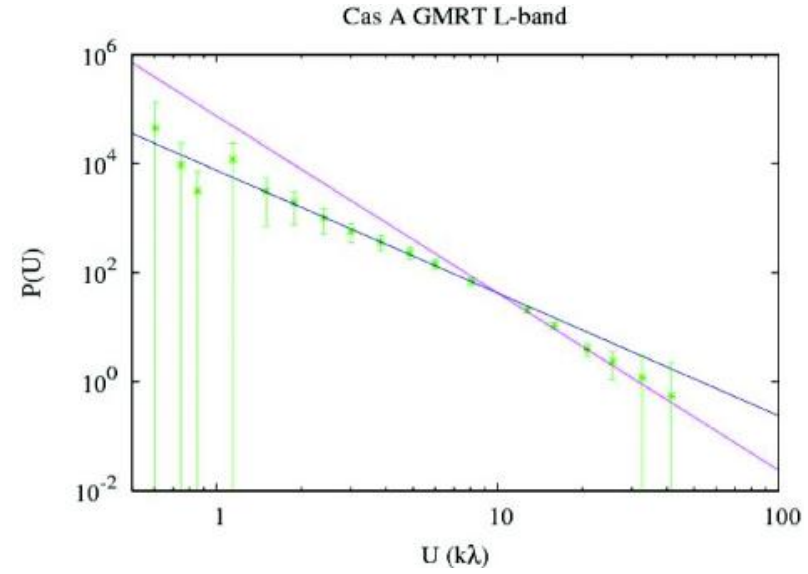

Fig. 7: Power spectrum of the synchrotron emission intensity fluctuations for Cas $\mathrm{A}$ at $1.4 \mathrm{GHz}$ estimated from the GMRT observation. The power law index changes from -2.22 to -3.23 at $U \gtrsim 10 \mathrm{k} \lambda$ dispersion with size of clouds. Similar studies for the molecular clouds were earlier reported by Larson (1981) and others. As mentioned before, for the dense CNM phase, $T_{s}$ is a good proxy for $T_{k}$ (see sub-sections titled "H I $21 \mathrm{~cm}$ radiation" and "Derived physical quantities" for details). So, from the H I emissionabsorption studies, one can estimate the non-thermal velocity dispersion $v_{\mathrm{NT}}^{2} \propto\left(T_{k, \max }-T_{k}\right) \approx\left(T_{k, \max }-\right.$ $T_{s}$ ). Most of the times, it is not straightforward to estimate the distance of the cloud, and hence the size, directly. But, if the thermal pressure $P=n k T_{k}$ is assumed to be more or less constant, then the typical length scale of a cloud

$$
L \sim N(\mathrm{HI}) / n \quad N(\mathrm{HI}) /\left(P / k T_{k}\right) \propto N(\mathrm{HI}) T_{s} .
$$

This reasoning was used to derive the scaling of the form $v_{N T} \sim L^{\beta}$ using the published millennium Arecibo 21-cm absorption-line survey results (Heiles and Troland, 2003a,b). The results are summarized in Fig. 8. Over almost two orders of magnitude length scale, the data is well-represented by $\beta=0.7 \pm 0.1$ (Roy et al., 2008). For $N(\mathrm{H} \mathrm{I}) T_{s} \gtrsim 10^{21.5} \mathrm{~cm}^{-2} \mathrm{~K}$, the spread is higher, plausibly due to systematic effects of the spectral and spatial resolution of the Arecibo telescope. The result is consistent with the $2 / 3$ scaling predicted by Kolmogorov (1941), who derived this relation by assuming that the dissipation rate is scale invariant over the inertial range. Note that this correlation, whether or not actually related to a

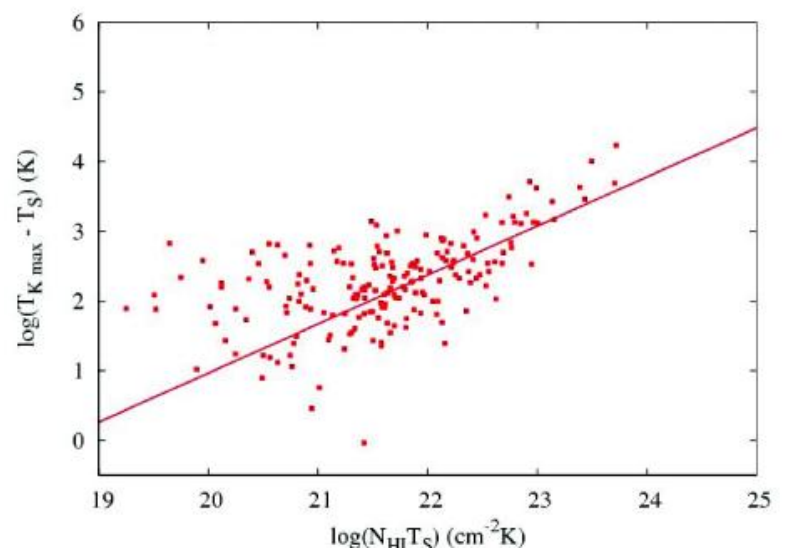

Fig. 8: Scaling of non-thermal line width with length scale derived from the millennium Arecibo $21 \mathrm{~cm}$ absorption-line survey results. A power law model with power law index $2 / 3$ is shown as a solid line 
Kolmogorov-like turbulence, can be used to estimate the non-thermal velocity dispersion and, in turn, the true kinetic temperature, taking it as a purely phenomenological model.

\section{Temperature of the Diffuse ISM}

In the classical model of multiphase ISM, the balance of heating and cooling rate leads to the two stable "phases" (CNM and WNM) of the neutral ISM. Gas at the intermediate kinetic temperature $\left(T_{k} \sim 500-\right.$ $5000 \mathrm{~K}$ ) will suffer from runaway heating or cooling to evolve into either the WNM or the CNM respectively. So, in this model, H I at intermediate temperature exists "only as a transient phenomenon", and does not contribute significantly to the total column density (Field et al., 1969; McKee and Ostriker, 1977; Wolfire et al., 2003). From an observational point of view, H I emission-absorption studies have shown the presence of narrow CNM components with high opacity, and with inferred $T_{k}$ in the expected range of $\lesssim 200 \mathrm{~K}$ (Radhakrishnan et al., 1972; Heiles and Troland, 2003a; Roy et al., 2006). The wide emission components with very little or no corresponding absorption are assumed to be originating from the WNM (see Fig. 5 and the related discussion in the "Derived physical quantities" sub-section). Due to their low optical depth, measurements of the WNM spin or kinetic temperature are rare (Carilli et al., 1998; Dwarakanath et al., 2002; Heiles, 2001; Begum et al., 2010; Murray et al., 2014). However, the thermal steady state scenario was considered to be the correct model broadly in agreement with the data.

Over the last few years, there were observational indications of the presence of a significant amount of $\mathrm{H} \mathrm{I}$ with temperature in the unstable range. These results seriously challenged the status of the standard model. Unfortunately, only a few of these measurements are from interferometric observations (Kanekar et al., 2003; Braun and Kanekar, 2005), and the rest comes from single dish observations (Millennium Arecibo $21 \mathrm{~cm}$ absorptionline survey; Heiles and Troland, 2003b). The main issue with such single dish observations is that the effect of the H I emission must be modelled accurately to derive the absorption spectra. This, and the contamination of the spectra by unwanted emission from the main beam may have significant systematic effects, and make the results less reliable. The other potential issue is that, for WNM, neither $T_{s}$ nor $T_{k, \max }$ is a reliable proxy for $T_{k}$; the non-thermal broadening may be significant, and also $T_{s}$ may considerably differ from $T_{k}$ (Liszt, 2001). Finally, in presence of CNM components along the line of sight, "self-absorption" of the background emission by the foreground components may make modelling the emission spectrum, if not impossible, really difficult. Even considering these possible observational shortcomings, the main result, that a large fraction of gas is in the "unstable" phase, has serious implications for the thermal steady state model.

It is important to note that Heiles and Troland (2003b) draw the conclusion of a significant fraction of unstable gas based on $T_{k, \max }$ derived from the observed emission line widths. It is possible to use a Kolmogorov-like scaling (see Fig. 8) to estimate the turbulent velocity dispersion, and derive the true temperature distribution. A reanalysis of the Heiles and Troland (2003b) data implementing this correction noticeably alters the distribution (Roy et al., 2009). Fig. 9 shows the main result - the distribution of $N(\mathrm{H} \mathrm{I})$ at different temperature ranges before and after correcting the non-thermal broadening. As

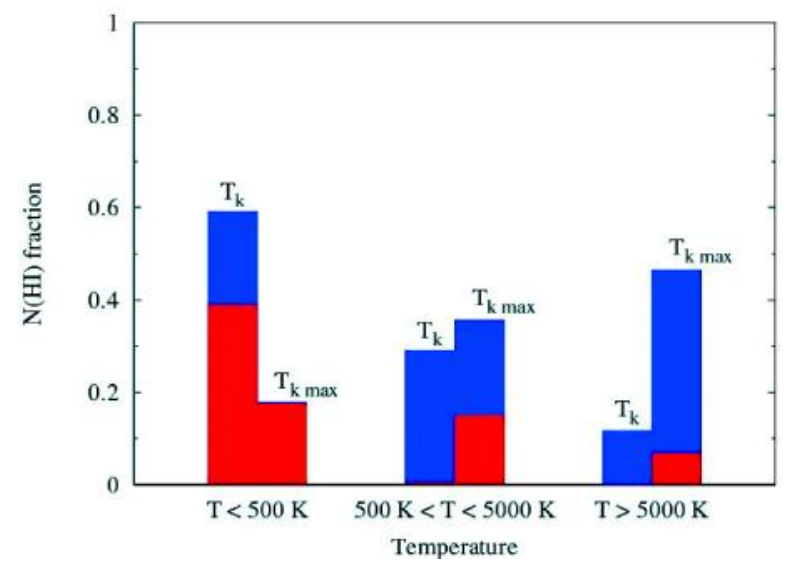

Fig. 9: Distribution of $H I$ in different phases based on temperatures computed without and with correcting the non-thermal broadening (bars marked with $T_{k, \max }$ and $T_{k}$ respectively). Red (blue) are $\mathrm{H} I$ emission components that are detected (not detected) in absorption. Note that the correction implies only a small amount of gas in the stable WNM phase; however, the unstable fraction remains almost unchanged 


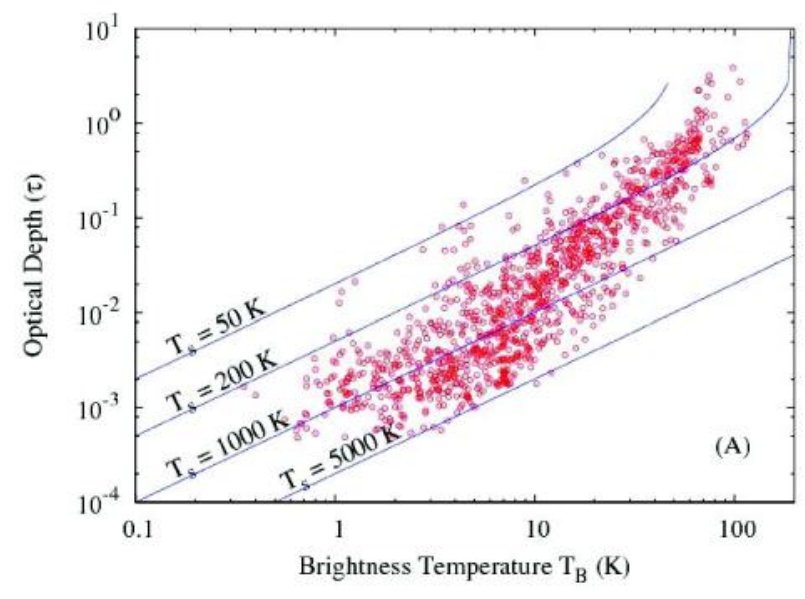

A

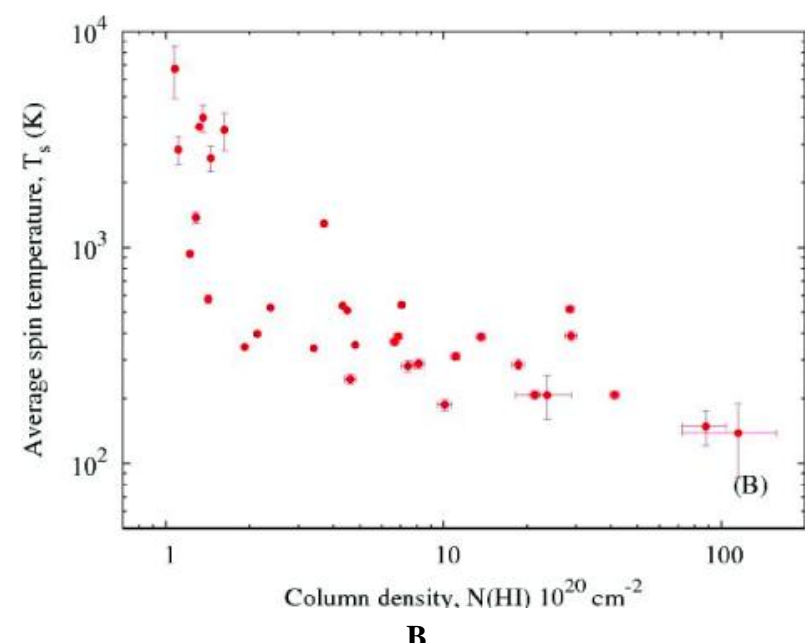

B

Fig. 10: (A): H I $21 \mathrm{~cm}$ optical depth at $1 \mathrm{~km} \mathrm{~s}^{-1}$ resolution from the absorption survey, and the corresponding $T_{B}$ from the LAB survey. Solid lines mark different $T_{s}$ values. Channels with intermediate $T_{s}$ may be a mix of cold and warm gas. (B): Integrated column density and average spin temperature for all 34 lines of sight. The high $T_{s}$ values imply presence of warm gas

expected, a lot of "warm" gases based on $T_{k, \max }$ are indeed just cold gases with significant non-thermal broadening. There is, in fact, very little $(\approx 10 \%) \mathrm{H} \mathrm{I}$ in the stable warm phase in this sample. However, even after this correction, a similar fraction of gas $(\approx 30 \%)$ remains in the unstable phase (Roy et al., 2009).

The fact that, due to various systematic effects, the single dish absorption spectra remains less reliable (particularly for weak and wide WNM components), prompted us to also start an interferometric survey with the GMRT and the WSRT. The main aim of this ongoing survey is to acquire high sensitivity, high spectral resolution Galactic H I $21 \mathrm{~cm}$ absorption towards a large number of background sources. A large sample, and a high sensitivity (RMS opacity $10^{-3}$ ) are necessary to draw a statistically significant conclusion about the WNM properties. So far, $\sim 50$ lines of sight have been observed (6- $8 \mathrm{hr}$ time per source). Details of the survey, the analysis procedure and results based on a brighter subsample of 34 sources, including two southern sources observed with the Australian Telescope Compact Array (ATCA), have been reported recently (Roy et al., 2013a,b). One example absorption spectrum from this survey is shown in Fig. 5.

From each of these lines of sight, using absorption spectra from this survey and the corresponding emission spectra from the LAB survey, $T_{s}$ values are computed at $\sim 1 \mathrm{~km} \mathrm{~s}^{-1}$ velocity resolution for each channel with $\geq 3 \sigma$ detection of $\mathrm{H} \mathrm{I}$ absorption. As shown in Fig. 10A, many channels have $T_{s}>200 \mathrm{~K}$, clearly an indication of a mix of cold and warm gases. The line of sight average $T_{s}$, plotted in Fig. 10B, also has values $>200 \mathrm{~K}$ for most of the cases. Based on $T_{s}$ at $1 \mathrm{~km} \mathrm{~s}^{-1}$ resolution, about $50 \%$ of the gas (by column density) has $T_{s}<200 \mathrm{~K}$ (i.e. channels with large cold gas fraction), and $>10 \%$ has $T_{s}<1000 \mathrm{~K}$ (possibly with large warm gas fraction). The rest, $\sim 40 \%$ of the gas, with $200 \mathrm{~K}<T_{s}$ $<1000 \mathrm{~K}$ is not necessarily "unstable" gas, but can be only a mix of CNM and WNM. However, even without Gaussian decomposition of the profiles, based on the average $T_{s}$ values and the sensitivity limit of the survey, one can conclude that the detected H I absorption is not entirely from cold gas with $T_{k}<200$ $\mathrm{K}$, and must also have contribution from (stable or unstable) warm gas (Kanekar et al., 2011; Roy et al., 2013a; Roy et al., 2013b). An upper limit of the CNM fraction was derived assuming that all cold gases have $T_{s}=200 \mathrm{~K}$. The median CNM fraction was found to be $f_{\mathrm{CNM}} \approx 0.52$ for the sample.

Finally, Gaussian decomposition of the absorption profiles was carried out, using the Levenberg-Marquardt $\chi^{2}$ minimization, to estimate $T_{k, \text { max }}$ of the individual clouds along the lines of sight. 
It was found that the number of components with $T_{k \text {,max }}>5000 \mathrm{~K}$ is very few (only seven in this range, and, considering the measurement uncertainty, possibly a few more out of a total 214 components). As the survey has adequate sensitivity to detect $\mathrm{H} \mathrm{I}$ in absorption even at this temperature range, one can put a strong constraint on the fraction of gas in the "classical" warm phase. Combining the facts (i) that only a few components have $T_{k, \max }$ in the range for stable WNM, (ii) that a significant fraction of gas can not be below the detection limit of the survey, and (iii) that the median $f_{\mathrm{CNM}}$ is at most about 0.5 , the lower limit of unstable gas fraction was found to be $\sim 0.3$. We conclude that, indeed, a significant fraction $(\gtrsim$ $30 \%$ ) of the gas is in the so-called unstable phase.

\section{Discussions and Conclusions}

Turbulence is an important ingredient of ISM physics in various regards (see Elmegreen and Scalo, 2004 and Scalo and Elmegreen, 2004, for comprehensive reviews on observations and implications of the ISM turbulence). The small scale structures of the ISM, originating from turbulence, seem to be ubiquitous not only in the Milky Way, but also in other galaxies (Westpfahl et al., 1999; Elmegreen et al., 2001; Dutta et al., 2009; Srianand et al., 2013). From the galaxy disk scale to molecular cloud, it affects the ISM dynamics and properties (Romeo et al., 2010; Shadmehri and Elmegreen, 2011; Hoffmann and Romeo, 2012). The results reported in this article, based on H I observations, show that both the density and velocity fluctuation statistics are consistent with a near-Kolmogorov turbulence in the diffuse ISM of our Galaxy. A working model is also proposed to estimate the non- thermal broadening of the line width and to derive the true kinetic temperature of the gas.

Apart from the H I $21 \mathrm{~cm}$ line, various other tracers at different wavelengths are also used to study turbulence in different phases of the ISM (Welty et al., 1994; Gibson, 2007; Boissé et al., 2013). However, neutral hydrogen, which is present almost everywhere in the Galaxy, can be used to probe turbulence over a wide range of scales, using multiple observational techniques (from single dish emission study to VLBI small scale absorption measurement).
For example, VLBI observations can constrain the density and the velocity power spectra at milliarcsec scales (e.g. Roy et al., 2012; Dutta et al., 2014). Constraining the turbulent behaviour of the ISM at the scale of VLBI observation can, in near future, address whether the same power law behaviour of the energy cascade is observed at pc and AU scales, and also constrain the energy dissipation scale and mechanism. It will also be useful to see, how the VLBI observations at AU scale compare with observations of tiny scale structures detected using multiwavelength observations of other tracers.

Recent Galactic H I observations are also questioning the classical thermal steady state model of the multiphase ISM. There is clear evidence that $\gtrsim 30 \%$ of the diffuse neutral gas is in the unstable phase. However, any effort of understanding the properties of the multiphase ISM should also take into account the possible effects of the turbulence. Observationally, one must get a handle on the turbulent velocity dispersion to accurately estimate the temperature, and to critically examine the validity of thermal steady state model. From theoretical consideration, on the other hand, turbulence may potentially play a role in creating and supporting gas at the intermediate temperature range. Recent numerical studies suggest that turbulence can explain the presence of some amount of gas in the unstable warm phase (e.g. Audit and Hennebelle, 2005; Audit and Hennebelle, 2010). These numerical simulations, however, still predict a large fraction of gas to be in the stable WNM phase, somewhat in contrast to the observational results. More recent hydrodynamic simulations by Kim et al. (2014) can explain some of the observational results of Roy et al. (2013a,b). It remains interesting to see whether more data and further numerical studies will result in a better agreement between the details of the observational results and the theoretical predictions.

\section{Acknowledgements}

The author thanks the Indian National Science Academy (INSA) and Professor Lakhotia, Editor-inChief, for giving him a chance of writing this article for the Proceedings of the INSA. The author is grateful 
to Aritra Basu for useful comments on an earlier version of the paper.

The author acknowledges his collaborators Somnath Bharadwaj, Robert Braun, Jayaram N. Chengalur, Prasun Dutta, Leshma Peedikakkandy, and Nissim Kanekar for their contribution, help and support in various projects which form the basis of this review article. The author acknowledges support from the Alexander von Humboldt Foundation, and also acknowledges support from the National Centre for Radio Astrophysics (NCRA) of the Tata Institute of Fundamental Research (TIFR) during his Ph.D., when a significant fraction of this work was done. Photograph of the GMRT antennas is provided by Arup Biswas, and those of the Effelsberg telescope and the LOFAR station are from Susmita Chakravorty.

\section{References}

Audit E and Hennebelle P (2005) Thermal condensation in a turbulent atomic hydrogen flow Astron Astrophys $\mathbf{4 3 3}$ $1-13$

Audit E and Hennebelle P (2010) On the structure of the turbulent interstellar clouds. Influence of the equation of state on the dynamics of 3D compressible flows Astron Astrophys 51112

Bajaja E, Arnal E M, Larrarte J J, Morras R, Pöppel W G L and Kalberla P M W (2005) A high sensitivity HI survey of the sky at $\delta \leq-25^{\circ}$. Final data release Astron Astrophys $440767-773$

Barnard E E (1919) On the dark markings of the sky, with a catalogue of 182 such objects Astrophys J 49 1-24

Begum A, Stanimirovic S, Goss W M, Heiles C, Pavkovich A S and Hennebelle P (2010) The Thermally Unstable Warm Neutral Medium: Key for Modeling the Interstellar Medium Astrophys J 725 1779-1785

Bharadwaj S and Sethi S K (2001) H I Fluctuations at Large Redshifts: I - Visibility correlation J Astrophys Astron 22 293-307

Boissé P, Federman S R, Pineau des Forêts G and Ritchey A M (2013) The au-scale structure in diffuse molecular gas towards $\zeta$ Persei Astron Astrophys $\mathbf{5 5 9} 7$

Braun R and Kanekar N (2005) Tiny H I clouds in the local ISM Astron Astrophys 436 L53-L56
The results reported here are based on data mainly from observations with the GMRT and the WSRT, and analysis carried out using the NRAO AIPS. The author thanks the staff of the GMRT and the WSRT who have made these observations possible. The GMRT is run by NCRA-TIFR. The WSRT is operated by ASTRON (the Netherlands Institute for Radio Astronomy), with support from the Netherlands Foundation for Scientific Research (NWO). The NRAO is a facility of the National Science Foundation (NSF) operated under cooperative agreement by Associated Universities, Inc. (AUI). Some results are also derived from the ATCA observations, the VLA archival data, the Leiden/Argentine/Bonn Galactic H I Survey data, and the millennium Arecibo 21-cm absorption-line survey data. This research has also made use of the NASA's Astrophysics Data System.

Brogan C L, Zauderer B A, Lazio T J, Goss W M, De Pree C D and Fasion M D (2005) Spatial and Temporal Variations in Small-Scale Galactic H I Structure toward 3C 138 Astron J 130 698-710

Burke B F and Graham-Smith F (2014) An Introduction to Radio Astronomy (3rd edition). Cambridge University Press (Cambridge)

Carilli C L, Dwarakanath K S and Goss W M (1998) Detection of H I 21 Centimeter Absorption by the Warm Neutral Medium Astrophys J Lett 502 L79-L83

Crovisier J and Dickey J M (1983) The spatial power spectrum of galactic neutral hydrogen from observations of the 21cm emission line Astron Astrophys 122 282-296

Deshpande AA, Dwarakanath K S and Goss W M (2000) Power Spectrum of the Density of Cold Atomic Gas in the Galaxy toward Cassiopeia A and Cygnus A Astrophys J 543 227234

Dickey J M and Lockman F J (1990) H I in the Galaxy Annu Rev Astron Astr 28 215-261

Dutta P, Begum A, Bharadwaj S and Chengalur J N (2009) A study of interstellar medium of dwarf galaxies using $\mathrm{H} \mathrm{I}$ power spectrum analysis Mon Not R Astron Soc 398 887897

Dutta P, Chengalur J N, Roy N, Goss W M, Arjunwadkar M, Minter A H, Brogan C L and Lazio T W J (2014) The structure function of Galactic H I opacity fluctuations on AU scales based on MERLIN, VLA and VLBA data Mon 
Not R Astron Soc 442 647-655

Dwarakanath K S, Carilli C L and Goss W M (2002) Detection of H I 21 Centimeter Line Absorption in the Warm Neutral Medium and in the Outer Arm of the Galaxy Astrophys $J$ $567940-946$

Elmegreen B G, Kim S and Staveley-Smith L (2001) A Fractal Analysis of the H I Emission from the Large Magellanic Cloud Astrophys J 548 749-769

Elmegreen B G and Scalo J (2004) Interstellar Turbulence I: Observations and Processes Annu Rev Astron Astr 42 211273

Ewen H I and Purcell E M (1951) Observation of a Line in the Galactic Radio Spectrum: Radiation from Galactic Hydrogen at 1,420 Mc./sec. Nature 168 356-358

Falgarone E, Puget J-L and Perault M (1992) The small-scale density and velocity structure of quiescent molecular clouds Astron Astrophys 257 715-730

Field G B (1958) Excitation of the Hydrogen 21-CM Line P IRE $46240-250$

Field G B (1965) Thermal Instability Astrophys $J 142$ 531-567

Field G B, Goldsmith D W and Habing H J (1969) Cosmic-Ray Heating of the Interstellar Gas Astrophys J Lett 155 L149L154

Frail D A, Weisberg J M, Cordes J M and Mathers C (1994) Probing the interstellar medium with pulsars on AU scales Astrophys J 436 144-151

Gibson S J (2007) Multiscale Structure in Dust Reflection and Cold HI Astr Soc P 365 59-64

Goldreich P and Sridhar S (1995) Toward a theory of interstellar turbulence. 2: Strong alfvenic turbulence Astrophys J 438 763-775

Green D A (1993) A power spectrum analysis of the angular scale of Galactic neutral hydrogen emission towards $\mathrm{L}=140$ deg, B = 0 deg Mon Not R Astron Soc 262 327-342

Hartmann D and Burton W B (1997) Atlas of Galactic Neutral Hydrogen. Cambridge University Press

Hartmann J (1904) Investigations on the spectrum and orbit of delta Orionis Astrophys $J 19$ 268-286

Heiles C (2001) New Temperatures of Diffuse Interstellar Gas: Thermally Unstable Gas Astrophys J 551 L105-L108

Heiles C and Troland T (2003a) The Millennium Arecibo 21 Centimeter Absorption-Line Survey. I. Techniques and Gaussian Fits Astrophys J Suppl S 145 329-354

Heiles C and Troland T (2003b) The Millennium Arecibo 21 Centimeter Absorption-Line Survey. II. Properties of the Warm and Cold Neutral Media Astrophys J 586 10671093
Heithausen A, Bensch F, Stutzki J, Falgarone E and Panis J F (1998) The IRAM key project: small-scale structure of pre-star forming regions. Combined mass spectra and scaling laws Astron Astrophys 331 L65-L68

Hill A S, Stinebring D R, Asplund C T, Berkwick D E, Everett W B and Hinkel N R (2005) Deflection of Pulsar Signal Reveals Compact Structures in the Galaxy Astrophys J Lett 619 L171-L174

Hoffmann V and Romeo A B (2012) The effect of ISM turbulence on the gravitational instability of galactic discs Mon Not $R$ Astron Soc 425 1511-1520

Iroshnikov P (1964) Turbulence of a Conducting Fluid in a Strong Magnetic Field Sov Astron 7 566-571

Jansky K G (1933) Radio Waves from Outside the Solar System Nature 132 66-66

Kalberla P M W, Burton W B, Hartmann D, Arnal E M, Bajaja E, Morras R and Pöppel W G L (2005) The Leiden/Argentine/ Bonn (LAB) Survey of Galactic H I. Final data release of the combined LDS and IAR surveys with improved strayradiation corrections Astron Astrophys 440 775-782

Kanekar N, Subrahmanyan R, Chengalur J N and Safouris V (2003) The temperature of the warm neutral medium in the Milky Way Mon Not R Astron Soc 346 L57-L61

Kanekar N, Braun R and Roy N (2011) An H I Column Density Threshold for Cold Gas Formation in the Galaxy Asrophys $J$ Lett 7375

Kim C G, Ostriker E C and Kim W-T (2014) Three-dimensional Hydrodynamic Simulations of Multiphase Galactic Disks with Star Formation Feedback. II. Synthetic H I $21 \mathrm{~cm}$ Line Observations Astrophys J 78613

Kolmogorov A (1941) The Local Structure of Turbulence in Incompressible Viscous Fluid for Very Large Reynolds' Numbers Dokl Akad Nauk SSSR + 30 301-305

Kraichnan R (1965) Inertial-Range Spectrum of Hydromagnetic Turbulence Phys Fluids 8 1385-1387

Kulkarni S R and Heiles C (1988) Neutral hydrogen and the diffuse interstellar medium. In: Galactic and Extragalactic Radio Astronomy (2nd edition) (Eds: Verschuur G and Kellerman K) pp 95-153, Springer-Verlag

Larson R B (1981) Turbulence and star formation in molecular clouds Mon Not R Astron Soc 194 809-826

Lazarian A and Pogosyan D (2000) Velocity Modification of H I Power Spectrum Astrophys J 537 720-748

Lee L C and Jokipii J R (1975) Strong scintillations in astrophysics. I - The Markov approximation, its validity and application to angular broadening Astrophys $J 196$ 695-707

Liszt H (2001) The spin temperature of warm interstellar H I 
Astron Astrophys 371 698-707

McKee C F and Ostriker J P (1977) A theory of the interstellar medium - Three components regulated by supernova explosions in an inhomogeneous substrate Astrophys $J$ 218 148-169

Mebold U, Winnberg A, Kalberia P M W and Goss W M (1982) An Effelsberg-Green Bank galactic H I absorption line survey. II - Results and interpretation Astron Astrophys $115223-241$

Murray C E, Lindner R R, Stanimirovic S, Goss W M, Heiles C, Dickey J, Pingel N M, Lawrence A, Jencson J, Babler B L and Hennebelle P (2014) Excitation Temperature of the Warm Neutral Medium as a New Probe of the Ly $\alpha$ Radiation Field Astrophys J Lett $\mathbf{7 8 1} 5$

Radhakrishnan V, Murray J D, Lockhart P and Whittle R P J (1972) The Parkes Survey of 21-Centimeter Absorption in Discrete-Source Spectra. II. Galactic 21-Centimeter Obser- vations in the Direction of 35 Extragalactic Sources Astrophys J Suppl S 24 15-47

Romeo A B, Burkert A and Agertz O (2010) A Toomre-like stability criterion for the clumpy and turbulent interstellar medium Mon Not R Astron Soc 407 1223-1230

Roy N, Chengalur J N, Srianand R (2006) A multiwavelength investigation of the temperature of the cold neutral medium Mon Not R Astron Soc 365 L1-L5

Roy N, Peedikakkandy L and Chengalur J N (2008) Turbulence measurements from $\mathrm{H}$ I absorption spectra Mon Not $R$ Astron Soc 387 L18-L22

Roy N, Dutta P, Bharadwaj S and Chengalur J N (2009) Magnetohydrodynamic turbulence in supernova remnants Mon Not R Astron Soc 393 L26-L30

Roy N, Chengalur J N, Dutta P and Bharadwaj S (2010) H I 21 cm opacity fluctuations power spectra towards Cassiopeia A Mon Not R Astron Soc 404 L45-L49

Roy N, Minter A H, Goss W M, Brogan C L and Lazio T J W (2012) Tiny Scale Opacity Fluctuations from VLBA, MERLIN, and VLA Observations of H I Absorption toward 3C 138 Astrophys $\mathbf{J 4 9} 5$

Roy N, Kanekar N, Braun R and Chengalur J N (2013a) The temperature of the diffuse $\mathrm{H}$ I in the Milky Way - I. High resolution H I-21 cm absorption studies Mon Not R Astron Soc 436 2352-2365

Roy N, Kanekar N and Chengalur J N (2013b) The temperature of the diffuse H I in the Milky Way - II. Gaussian decomposition of the H I-21 cm absorption spectra Mon Not R Astron Soc 436 2366-2385

Scalo J and Elmegreen B G (2004) Interstellar Turbulence II: Implications and Effects Annu Rev Astron Astr 42 275316

Shadmehri M and Elmegreen B G (2011) Mass functions in fractal clouds: the role of cloud structure in the stellar initial mass function Mon Not R Astron Soc 410 788-804

Srianand R, Gupta N, Rahmani H, Momjian E, Petitjean P and Noterdaeme P (2013) Parsec- scale structures and diffuse bands in a translucent interstellar medium at $z \simeq 0.079$, Mon Not R Astron Soc 428 2198-2206

Swarup G, Ananthakrishnan S, Kapahi V K, Rao A P, Subrahmanya C R and Kulkarni V K (1991) The Giant Metre-Wave Radio Telescope Curr Sci India 60 95-105

Thompsom A R, Moran J M and Swenson G W Jr (2001) Interferometry and Synthesis in Radio Astronomy (2nd edition). Wiley-Interscience

van de Hulst H C (1945) Radio Waves from Space: Origin of Radiowaves Nederlands Tijdschrift voor Natuurkunde 11 210-221

Welty D E, Hobbs L M and Kulkarni V P (1994) A high-resolution survey of interstellar Na I D1 lines Astrophys J 436 152175

Westpfahl D J, Coleman P H, Alexander J and Tongue T (1999) The Geometry of the H I of Several Members of the M81 Group: The H I Is Fractal Astron J 117 868-880

Wilson T L, Rohlfs K and Hüttemeister S (2013) Tools of Radio Astronomy (6th edition). Springer-Verlag

Wolfire M G, Hollenbach D, McKee C F, Tielens A G G M and Bakes E L O (1995) The neutral atomic phases of the interstellar medium Astrophys J 443 152-168

Wolfire M G, McKee C F, Hollenbach D amd Tielens A G G M (2003) Neutral Atomic Phases of the Interstellar Medium in the Galaxy Astrophys J 587 278-311. 